\title{
Hierarchical Organization of Human Cortical Networks in Health and Schizophrenia
}

\author{
Danielle S. Bassett, ${ }^{1,3,4}$ Edward Bullmore, ${ }^{3,5 *}$ Beth A. Verchinski, ${ }^{2}$ Venkata S. Mattay, ${ }^{2}$ Daniel R. Weinberger, ${ }^{1}$ and \\ Andreas Meyer-Lindenberg ${ }^{1,6 *}$ \\ ${ }^{1}$ Clinical Brain Disorders Branch, Genes Cognition and Psychosis Program and 2Neuroimaging Core Facility, National Institute of Mental Health, National \\ Institutes of Health, Bethesda, Maryland 20892, ${ }^{3}$ Brain Mapping Unit, Department of Psychiatry, Addenbrooke's Hospital, ${ }^{4}$ Biological Soft Systems Sector, \\ Department of Physics, and ${ }^{5}$ Behavioral and Clinical Neurosciences Institute, University of Cambridge, Cambridge CB2 1TN, United Kingdom, and ${ }^{6} \mathrm{Central}$ \\ Institute for Mental Health, D-68072 Mannheim, Germany
}

The complex organization of connectivity in the human brain is incompletely understood. Recently, topological measures based on graph theory have provided a new approach to quantify large-scale cortical networks. These methods have been applied to anatomical connectivity data on nonhuman species, and cortical networks have been shown to have small-world topology, associated with high local and global efficiency of information transfer. Anatomical networks derived from cortical thickness measurements have shown the same organizational properties of the healthy human brain, consistent with similar results reported in functional networks derived from resting state functional magnetic resonance imaging (MRI) and magnetoencephalographic data. Here we show, using anatomical networks derived from analysis of inter-regional covariation of gray matter volume in MRI data on 259 healthy volunteers, that classical divisions of cortex (multimodal, unimodal, and transmodal) have some distinct topological attributes. Although all cortical divisions shared nonrandom properties of small-worldness and efficient wiring (short mean Euclidean distance between connected regions), the multimodal network had a hierarchical organization, dominated by frontal hubs with low clustering, whereas the transmodal network was assortative. Moreover, in a sample of 203 people with schizophrenia, multimodal network organization was abnormal, as indicated by reduced hierarchy, the loss of frontal and the emergence of nonfrontal hubs, and increased connection distance. We propose that the topological differences between divisions of normal cortex may represent the outcome of different growth processes for multimodal and transmodal networks and that neurodevelopmental abnormalities in schizophrenia specifically impact multimodal cortical organization.

Key words: anatomy; network; hierarchy; systems; MRI; schizophrenia; neurodevelopment

\section{Introduction}

A concise quantification of the extraordinary complexity of the extensively interconnected human brain has long been elusive if not intractable. However, topological measures based on graph theory (Watts and Strogatz, 1998; Latora and Marchiori, 2001; Newman, 2002) have recently provided new approaches to the investigation of complex networks. Following the seminal descriptions of cortical networks, based on tract tracing data that directly define anatomical connections between regions (Young, 1992), graph theoretical metrics were applied to quantification of nonhuman cortical connectivity matrices (Hilgetag et al., 2000; Stephan et al., 2000; Sporns et al., 2000) [for review, see Bassett

\footnotetext{
Received May 2, 2008; revised June 27, 2008; accepted July 21, 2008.

This work was supported by a Human Brain Project Grant from the National Institute of Biomedical Imaging and Bioengineering and the National Institute of Mental Health and by the Intramural Research Program of the National Institutes of Health, National Institute of Mental Health. D.S.B. was supported by the National Institutes of Health Graduate Partnerships Program. We thank Sophie Achard for useful discussions regarding network statistics.

${ }^{*}$ E.B. and A.M.-L. contributed equally to this work.

Correspondence should be addressed to Dr. Edward Bullmore, Brain Mapping Unit, Department of Psychiatry, Addenbrooke's Hospital, Hills Road, Cambridge CB2 2QQ, UK. E-mail: etb23@cam.ac.uk.

D01:10.1523/JNEUROSCI.1929-08.2008

Copyright $\odot 2008$ Society for Neuroscience $\quad$ 0270-6474/08/289239-10\$15.00/0
}

and Bullmore (2006)]. Thus, it has been established that anatomical networks in both the cat and the macaque monkey have the typical "small-world" combination of high clustering of local connections with a short minimum path length between any pair of regions (Hilgetag et al., 2000; Sporns et al., 2002; Sakata et al., 2005); they also tend to minimize wiring length (Chen et al., 2006).

Comparable information on human anatomical networks, for which tract tracing data are not available, can be inferred from magnetic resonance imaging (MRI) data. For example, cortical thickness measurements are known to be strongly correlated between regions that are axonally connected (Lerch et al., 2006), perhaps because connectivity confers a mutually trophic effect on growth of connected regions (Wright et al., 1999; Pezawas et al., 2005). Thus, a whole-brain anatomical network can be abstracted from human MRI data by compiling a matrix of correlations in cortical thickness (or volume) between all pairs of regions in some parcellation scheme and then applying a threshold to create a graph representing strong (suprathreshold) correlations as connections between regions (see Fig. 1). This approach was used previously to confirm that human neuroanatomical networks also have small-world properties, that the most highly connected 
regions, or "hubs," of the network tend to be areas of association cortex, and that the network has a modular community structure (Chen et al., 2008; He et al., 2008). Importantly, many of these results are consistent with recent graph theoretical analyses of human brain functional networks in a no-task or "resting" state (Salvador et al., 2005; Achard et al., 2006; Bassett et al., 2006; Achard and Bullmore, 2007), indicating a degree of topological isomorphism between whole-brain structural and functional networks.

The classical divisions of cerebral cortex (unimodal, multimodal, and transmodal), originally defined by regional cytoarchitectonic characteristics, are known to support very different functions (Mesulam, 1998). Although it seems reasonable to expect that these varied functions are reflected in division-specific organization of anatomical connections between regions, no data are currently available to test this hypothesis. One of the principal goals of this study was therefore to assess the commonalities and differences in network organization of the principal human cortical divisions. To do this, we used several topological measures (including hierarchy and degree correlation, as formally defined in Materials and Methods) and an estimate of the spatial separation between connected regional nodes (connection distance).

Moreover, if topological measures of anatomical network organization are functionally and/or pathophysiologically relevant, we might expect them to be impacted by neuropsychiatric disorders, such as schizophrenia, that are associated with abnormal cognitive function and measures suggestive of disordered anatomical connectivity (Volkow et al., 1988; Weinberger et al., 1992; Bullmore et al., 1997; Meyer-Lindenberg et al., 2001). Another major goal of this study was therefore to investigate whether topological and distance metrics of anatomical network organization were significantly abnormal in people with schizophrenia.

\section{Materials and Methods \\ Sample}

Two hundred three patients with schizophrenia and related spectrum disorders (diagnosed according to Diagnostic and Statistical Manual of Mental Disorders $I V$ criteria) and 259 healthy volunteers were recruited as part of the Clinical Brain Disorders Branch/National Institute of Mental Health Genetic Study of Schizophrenia [National Institutes of Health Study Grant NCT 00001486, Daniel R. Weinberger, principal investigator] (supplemental Table $1 A-D$, available at www.jneurosci.org as supplemental material). Additional details of the sampling protocol and full exclusion criteria are described by Egan et al. (2000). In the patient group, $81 \%$ had a diagnosis of schizophrenia, $12 \%$ had schizoaffective disorder, and $7 \%$ had psychosis not otherwise specified.

The two groups were matched for age, but the schizophrenia group included more males and had lower mean intelligence quotient and years of education than the volunteer group. All the patients were receiving antipsychotic drugs and other medication at the time of study; none of the healthy volunteers were taking psychoactive medication (Egan et al., 2000).

\section{Acquisition and preprocessing of MRI data}

A high-resolution magnetic resonance image of brain anatomy was acquired from each participant using a GE Signa system (GE Healthcare) operating at $1.5 \mathrm{~T}$ at the National Institute of Mental Health. A threedimensional volume of T1-weighted data, comprising 124 sections in the sagittal plane, was acquired using a spoiled gradient recall sequence with the following parameters: repetition time, $24 \mathrm{~ms}$; echo time, $5 \mathrm{~ms}$; number of excitations, 1 ; flip angle, $45^{\circ}$; matrix size, $256 \times 256$; field of view, $24 \times 24 \mathrm{~cm}$; voxel resolution (in $x, y, z$ dimensions), $0.975 \times 0.975 \times 1.5$ $\mathrm{mm}^{3}$. Participants were scanned in random order with respect to diagnosis. Quality control procedures were as described previously (Egan et al., 2000).
Table 1. Areas allocated to transmodal, unimodal, and multimodal systems

\begin{tabular}{|c|c|c|}
\hline Transmodal & Unimodal & Multimodal \\
\hline ObF (11) & PSs (1) & FEF (8) \\
\hline ObF (12) & PSs (2) & DLpF (9) \\
\hline Ins (13) & PSs (3) & $\mathrm{FPo}(10)$ \\
\hline Ins (14) & PrM (4) & V3 (19) \\
\hline Ins (15) & SsA (5) & $\ln T(20)$ \\
\hline Ins (16) & SuM (6) & MiT (21) \\
\hline VPC (23) & SsA (7) & SuT (22) \\
\hline VAC (24) & $\operatorname{FEF}(8)$ & $\mathrm{PHg}$ (36) \\
\hline $\operatorname{SbG}(25)$ & PVi (17) & $\operatorname{lnT}(37)$ \\
\hline $\operatorname{EcS}(26)$ & ViA (18) & AnG (39) \\
\hline RPH (27) & $\operatorname{PrA}(41)$ & $\mathrm{SmG}(40)$ \\
\hline PER (28) & AuA (42) & $\mathrm{SbC}(43)$ \\
\hline $\operatorname{ReS}(29)$ & $\mathrm{SbC}(43)$ & $p \operatorname{Tr}(44)$ \\
\hline Cin $(30)$ & & p0p (45) \\
\hline DPC (31) & & DLpF (46) \\
\hline DAC (32) & & $\operatorname{lnpF}(47)$ \\
\hline
\end{tabular}

ACC (33)

$\operatorname{AER}(34)$

$\operatorname{PeR}(35)$

TPo (38)

$\mathrm{RSb}$ (48)

Regional categorizations were based on previous work (Mesulam, 1998). Brodmann's areas are shown in parentheses. ObF, Orbitofrontal; Ins, insula; VPC, ventral posterior cingulate; $V A C$, ventral anterior cingulate; $S b G$, subgenual; ECS, ectosplenial; RPH, rostral parahippocampus; PER, posterior entorhinal; ReS, retrosplenial; Cin, cingulate; DPC, dorsal posterior cingulate; DAC, dorsal anterior cingulate; ACC, anterior cingulate; AER, anterior entorhinal; PeR, perirhinal; $T P$, temporopolar; RSb, retrosubicular; $P S S$, primary somatosensory; PrM, primary motor; SsA, somatosensory association; SuM, supplementary motor; FEF, frontal eye field; PVi, primary visual; ViA, visual association; PrA, primary auditory; AuA, auditory association; SbC, subcentral area; DLpF, dorsolateral prefrontal cortex; FPO, frontopolar; InT, inferior temporal: MiT, middle temporal; SuT, superior temporal; $\mathrm{PHg}$, parahippocampal gyrus; InT, inferior temporal; $\mathrm{AnG}$, angular gyrus; $\mathrm{SmG}$, supramarginal gyrus; $\mathrm{SbC}$, subcentral area; $\mathrm{pTr}$, pars triangularis; $\mathrm{p} 0 \mathrm{p}$ pars opercularis; InpF, inferior prefrontal.

Overall, the preprocessing pipeline included the following steps: inhomogeneity correction; stripping of skull and other noncerebral tissues; probabilistic tissue classification of gray matter, white matter, and CSF density at each voxel; nonlinear normalization of each image with a custom T1-weighted template image [based on a previous sample of 171 healthy volunteers scanned using the same system and sequence (Pezawas et al., 2004)]; modulation of the gray matter densities in the normalized images by the determinant of the Jacobian of the normalization function to provide an estimate of gray matter volume at each voxel (Good et al., 2001). These operations were implemented using SPM2 software (Statistical Parametric Mapping, http://www. fil.ion.ucl.ac.uk/spm/).

We then estimated the total gray matter volume for each of $n=104$ brain regions in each participant. The 104 regions comprised 48 cortical regions in each hemisphere, corresponding approximately to classical Brodmann areas (BAs), as well as the amygdala, the hippocampus, the striatum, and the thalamus bilaterally. Regions were defined anatomically by previous template images (Pick-Atlas, Advanced Neuroscience Imaging Research Core, http://www.fmri.wfubmc.edu; MRIcro, http:// www.sph.s.c.edu/comd/rorden/mricro.html) that were registered with the gray matter volume maps by an affine registration. Cortical areas were further categorized into transmodal $T$, unimodal $U$, or multimodal $M$ divisions using previously published criteria (Mesulam, 1998). The transmodal division comprised 42 regions, the unimodal division comprised 28 regions, and the multimodal division comprised 32 regions (Table 1).

We used regression to model the effects of age (linear, quadratic, and cubic functions), gender, and total gray matter volume on the full set of individual measurements at each region. The residuals of this regression, or corrected gray matter volume estimates, therefore represent regional volumes corrected for age, gender, and total gray matter volume and were the substrate for additional analysis.

\section{Inter-regional correlations and anatomical networks}

We used partial correlation as the metric of association between corrected gray matter volume estimates for each possible pair of regions in 
each group separately. For each pair of regions $i$ and $j$, we computed the correlation in corrected gray matter volume over subjects $\rho_{i, j}$ after removing the effects of all other regions $m \neq(i, j)$. This resulted in a pair of $\{104 \times 104\}$ partial correlation matrices, each within-group matrix representing the specific associations in corrected gray matter volume between each of the 5356 possible pairs of regions.

From each partial correlation matrix $R$, we derived a binary adjacency matrix $A$, the $a^{i, j}$ elements of which are zero if $\rho_{|i, j|}<\tau$ and unity if $\rho_{|i, j|}$ $\geq \tau$, where $0<\tau<1$ is an arbitrary threshold. The adjacency matrix can in turn be represented as an undirected graph $G$, where a line or edge is drawn between two regional nodes if the partial correlation between them exceeds the threshold.

Rather than restricting our analysis to a set of graphs obtained by applying a single threshold value to the partial correlation matrices, we systematically explored the properties of the graphs over a range of thresholds. When $\tau \rightarrow 1$, the number of edges in the graph $E$ will monotonically decrease toward its minimum value $(0)$; when $\tau \rightarrow 0$, the number of edges will monotonically increase toward its maximum value $\left(E_{\max }=5356\right)$. The number of edges in a graph, divided by the maximum possible number of edges, can be used as a simple measure of the threshold-dependent cost of the graph (Latora and Marchiori, 2001; Achard and Bullmore, 2007): $0<K(\tau)=E(\tau) / E_{\max }<1$. Thus, it is possible to represent various measures of network organization within each group as a function of cost and to compare topological and anatomical properties of the graphs between groups while ensuring that the number of edges is the same for each group over the range of thresholds considered (Bollobás, 1985).

\section{Overview of network analysis}

We investigated network architecture at global, divisional, and regional scales. Analysis of cortical divisions, by definition, only included cortical regions, whereas analysis at global and regional scales also included subcortical regions. Global network architecture was quantified in terms of small-worldness and degree distribution of the whole-brain network. Divisional architecture was characterized in terms of small-worldness, degree distribution, hierarchy $(\beta)$, degree correlation $(r)$, and connection distance $(d)$. Regional properties were described in terms of degree $(k)$, clustering $(C)$, and several measures of centrality (e.g., betweenness centrality, $B_{c}$ ). The computation of these metrics requires binary graphs, or adjacency matrices, obtained by thresholding the partial correlation matrices. Thus, we first established the range of thresholds that yielded fully connected, small-world graphs. We then averaged the divisional and regional metrics over this small-world regime and compared the results, when appropriate, to null distributions obtained from randomly connected graphs. Furthermore, using permutation tests, we looked for significant differences in divisional and regional metrics between the normal and schizophrenic groups.

\section{Topological and distance metrics}

Here we provide brief, formal definitions of each of the metrics used in this study.

Degree, path length, clustering, and small-worldness. The degree of a node, $k$, is simply the number of edges that connect it to the rest of the network: highly connected nodes have large degree. The degree distribution represents the probability of a given degree over all nodes in the network.

The minimum path length between two nodes in a graph, $L_{i, j}$, is the smallest number of edges that must be traversed to make a connection between them. If $L_{i, j}=1$, the two nodes are nearest neighbors, directly connected to each other by a single edge. The characteristic path length, or average of $L_{i, j}$ over all possible pairs, is thus a measure of the global connectivity of the network.

The clustering coefficient, $C$, is defined as follows:

$$
C=\frac{1}{N_{G i}\left(N_{C i}-1\right)} \sum_{j, k \in G_{i}} \frac{1}{L_{j, k}},
$$

where $G_{i}$ is the subgraph of nodes and edges connected to node $i$, and $L_{j, k}$ is the minimum path length between nodes $j$ and $k$ in the subgraph. Thus, clustering is a measure of the local connectivity of a regional node.
Small-worldness, $\sigma$, of a complex network is defined as high clustering $C$ compared with the clustering of a comparable random graph $C_{R}$ but characteristic path length approximately the same as in a comparable random graph $L_{R}$ (Watts and Strogatz, 1998). This can be summarized by the scalar $\sigma=\frac{C / C_{R}}{L / L_{R}}$ (Humphries et al., 2006), which will be greater than unity for a small-world network. We estimated the small-worldness of the whole-brain network at each cost by sampling 100 random graphs (with the same number of nodes and degree distribution as the brain networks) and using the resulting estimates of $L_{R}$ and $C_{R}$ to estimate the distribution of $\sigma$. We defined the upper limit of the small-world regime as the highest cost $(K=0.25)$ at which the minimum value of $\sigma$ was greater than 1 .

Hierarchy. The hierarchical structure of the networks was quantified by the $\beta$ coefficient, which is a parameter of the power-law relationship between clustering $C$ and degree $k$ of the nodes in the network (Ravasz and Barabasí, 2003): $\mathrm{C} \sim \mathrm{k}^{-\beta}$. We estimated $\beta$ by fitting a linear regression line to the plot of $\log (C)$ versus $\log (k)$ for the network at a given cost. To estimate hierarchy coefficients for the multimodal, unimodal, and transmodal divisions of cortex, we used the same regression model but fitted it separately to the regional data representative of each division. A large positive value of $\beta$ means that the hubs of the network have high degree (total connectivity) but low clustering (local connectivity), indicating that they are connected predominantly to nodes not otherwise connected to each other.

Assortativity. The degree correlation, $r$, is a measure of the correlation between the degree of a node and the mean degree of its nearest neighbors (Newman, 2002). This parameter can be adapted to determine the assortativity of subnetworks by considering only the subset of edges that connect to any of the nodes within the subnetwork. A positive value of degree correlation, $r>0$, indicates that nodes are likely to be connected to other nodes with the same degree and therefore that the high degree nodes or hubs of the network are likely to be connected to each other. This is characteristic of an assortative network. If the degree correlation is negative, $r<0$, this implies that the hubs of the network are not connected to each other, and this is characteristic of a disassortative network.

Connection distance. The connection distance of an edge, $d_{i, j}$, was estimated by the Euclidean distance between the centroids of the connected regions $i$ and $j$ in standard stereotactic space. The mean connection distance, $d$, was defined as the average connection distance over all edges in a network. Thus, connection distance differs from the other, topological and dimensionless metrics we used in that it represents a spatial or topographic property of the network and has units of millimeters.

Centrality and identification of network hubs. Several different metrics are available to identify the regional hubs of a network. As already noted, high degree is one such criterion; however, various measures of centrality have also been advocated for this purpose (Sporns et al., 2007). We used four measures to identify regional hubs: degree $k$, betweenness $B_{c}$, closeness $C_{c}$, and eigenvector centrality $E_{c}$. A given region was defined as a hub if its value for one or more of these measures was at least 2 SDs greater than the mean over all regional nodes in the network.

The betweenness centrality for the $i$ th region in a graph $G$ is defined as follows:

$$
B_{c}(i)=\sum_{j \neq m \neq i \in G} \frac{\sigma_{j, m}(i)}{\sigma_{j, m}},
$$

where $\sigma_{j, m}$ is the number of shortest geodesic paths between regions $j$ and $m$, and $\sigma_{j, m}(i)$ is the number of shortest geodesic paths between $j$ and $m$, which pass through node $i$.

The closeness centrality is defined as follows:

$$
C_{c}(i)=\frac{1}{\sum_{j \in G} L_{i, j}},
$$

where $j$ denotes one of the nodes accessible from the index node $i$, and $L_{i, j}$ is the shortest path between nodes $i$ and $j$.

The eigenvector centrality of the $i$ th node is the $i$ th component of the 

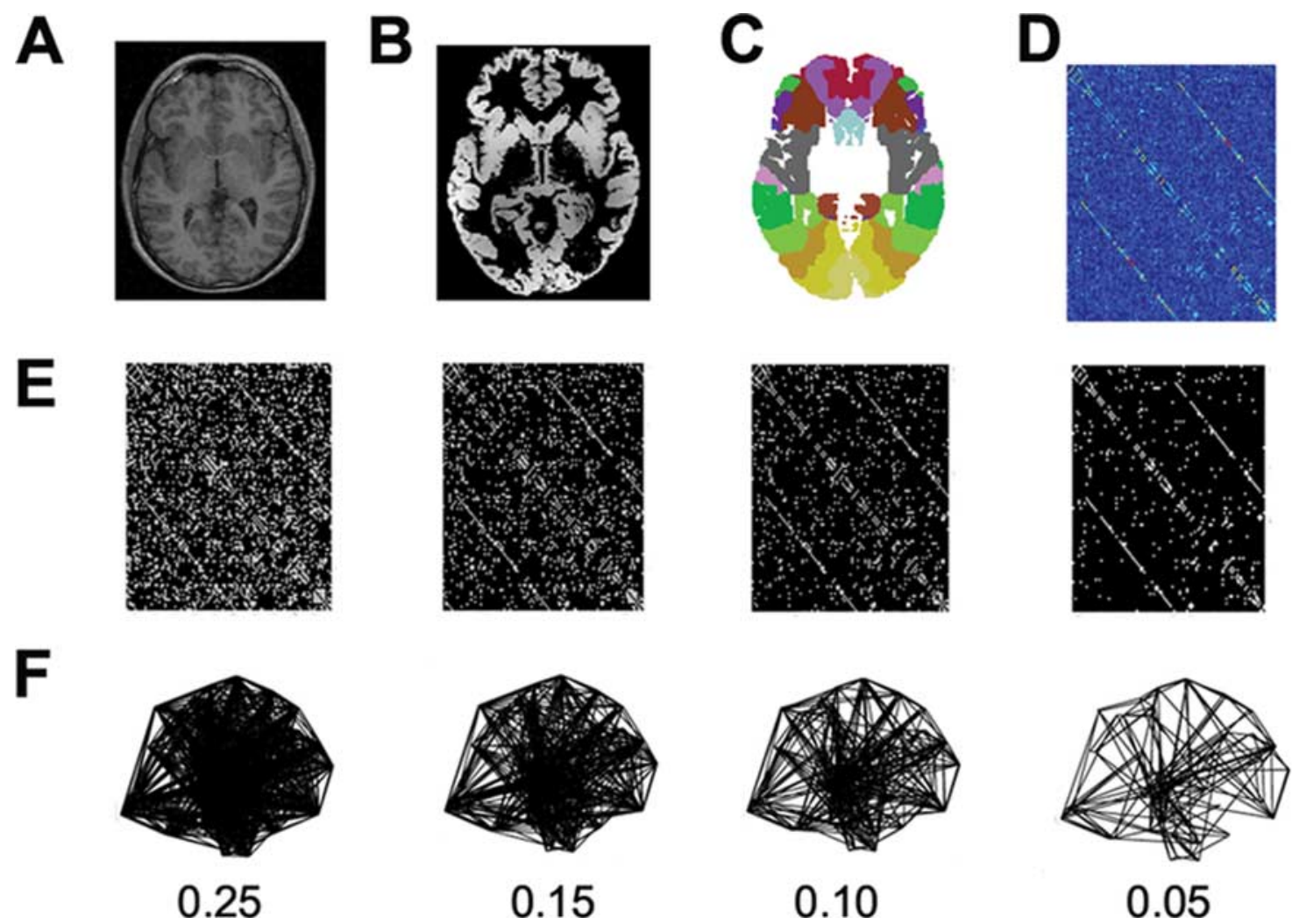

Figure 1. Schematic of procedure to construct human whole-brain anatomical networks. Top row, Structural MR images $(\boldsymbol{A})$ are segmented by a tissue classification algorithm to produce maps of gray matter $(\boldsymbol{B})$ that are then multiplied by a regionally parcellated template image $(\boldsymbol{C})$ to estimate regional gray matter volume in each of 104 brain regions. The partial correlation of regional gray matter volume is estimated for each possible pair of regions and compiled in a $\{104 \times 104\}$ inter-regional partial correlation matrix (D). Middle row, Various thresholds can be applied to generate adjacency matrices of variable sparseness from the partial correlation matrix (E). Bottom row, The adjacency matrices are visualized as undirected graphs or networks by plotting each region as a node in anatomical space (using the $x$ and $z$ coordinates of the regional centroid in Montreal Neurological Institute space) and drawing an edge between regions that have strongly correlated gray matter densities (non-zero elements in the adjacency matrix) $(\boldsymbol{F})$. Results are shown for networks with costs in the range $0.25 \geq K \geq 0.05$.

eigenvector of the adjacency matrix $A$ associated with the largest eigenvalue.

\section{Statistical analysis}

We compared the observed values of hierarchy $\beta$, degree correlation $r$, and connection distance $d$ in cortical networks to their distributions in comparable random graphs with the same number of nodes and degree distributions. At each cost in the small-world regime, we sampled 1000 random graphs and estimated the mean and SD of each parameter so that we could then calculate a $Z$-score for the corresponding parameter estimated in the cortical network. For example, the $Z$-score for the hierarchy coefficient of the multimodal network thresholded at arbitrary cost was thus defined as follows:

$$
Z(\mathcal{M})=\frac{\beta_{\mu}-\overline{\beta_{R}}}{S D\left(\beta_{R}\right)},
$$

where $\beta_{R}$ denotes the hierarchy coefficient of the comparable random graph. Maximum $Z$-scores, and $Z$-scores averaged over all costs in the small-world regime, i.e., $0.15 \leq K \leq 0.25$, were used to summarize the topological properties of the cortical networks compared with random networks.

To investigate topological and distance parameter differences between the healthy volunteers and the people with schizophrenia, we used a permutation test. The regional gray matter volume estimates for each participant were randomly reassigned to one of two groups comprising the same number of subjects as the volunteer (259) or schizophrenia (203) groups. The partial correlation matrix was then estimated and thresholded; the graph parameters were calculated at each cost in the small-world regime, and the between-group difference in all parameters was calculated for each permutation. This procedure was repeated 5000 times to sample the permutation distributions of all parameter differences under the null hypothesis that observed topological differences were not determined by true group membership. The one-tailed $p$ value was then calculated as the proportion of entries in the corresponding permutation distribution that were greater than (or smaller than) the observed between-group difference.

All topological and statistical operations were implemented using Statistica (version 6.0; StatSoft) or Matlab (version 2007a; MathWorks) software. The networks in Figure 4 were created using Pajek software (http://pajek.imfm.si/doku.php?id=download).

\section{Results}

\section{Defining a small-world cost regime}

The topological properties of the networks derived by thresholding the matrix of inter-regional correlations in gray matter volume will depend on the choice of threshold value. If the threshold is high and the number of edges is low, the network will be sparsely connected and some regional nodes may be disconnected; if the threshold is low and the number of edges is high, the network will be more densely connected but will also have a random topology (Fig. 1). Our first effort was therefore to define a range of thresholds that yielded fully connected networks with a small-world topology. We found that networks were fully connected when the cost of the network $K$, defined as the number of edges divided by the maximum possible number of edges, was $>0.15$. For each fully connected network, we calculated the small-world scalar $\sigma$, which provides a convenient summary of the small-worldness of a network compared with equally sized random graphs: if $\sigma \gg 1$, the network in question is small-world. 


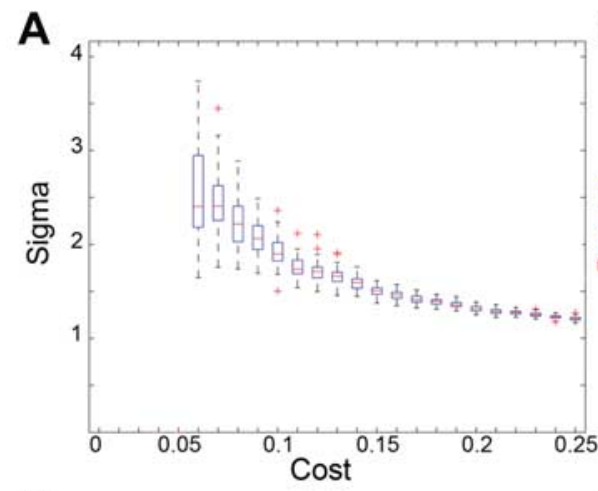

D

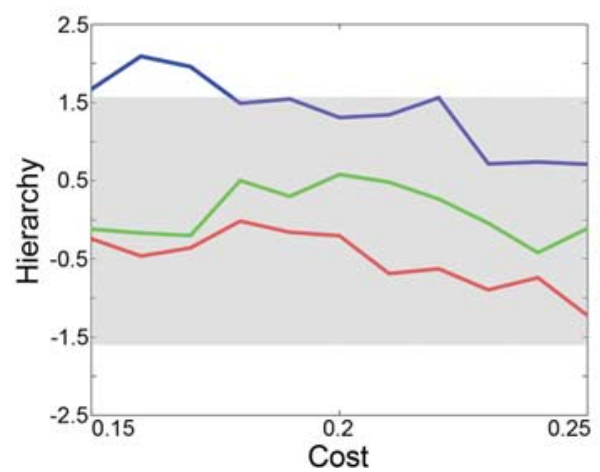

B

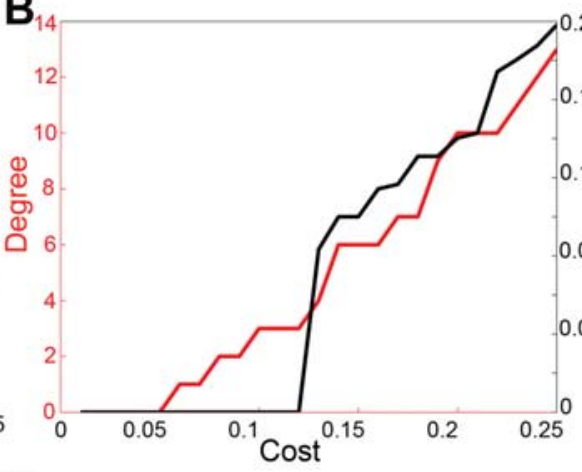

E

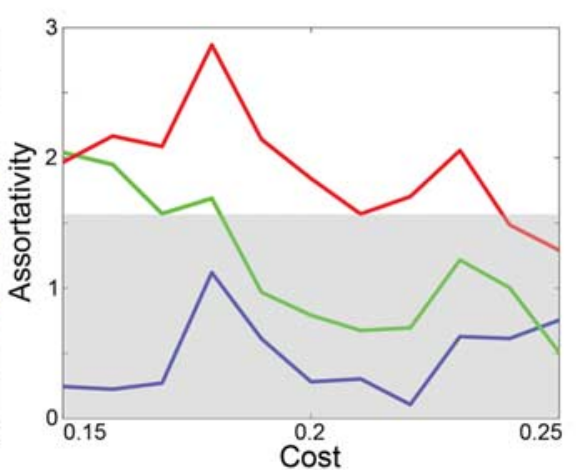

C

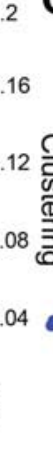

F
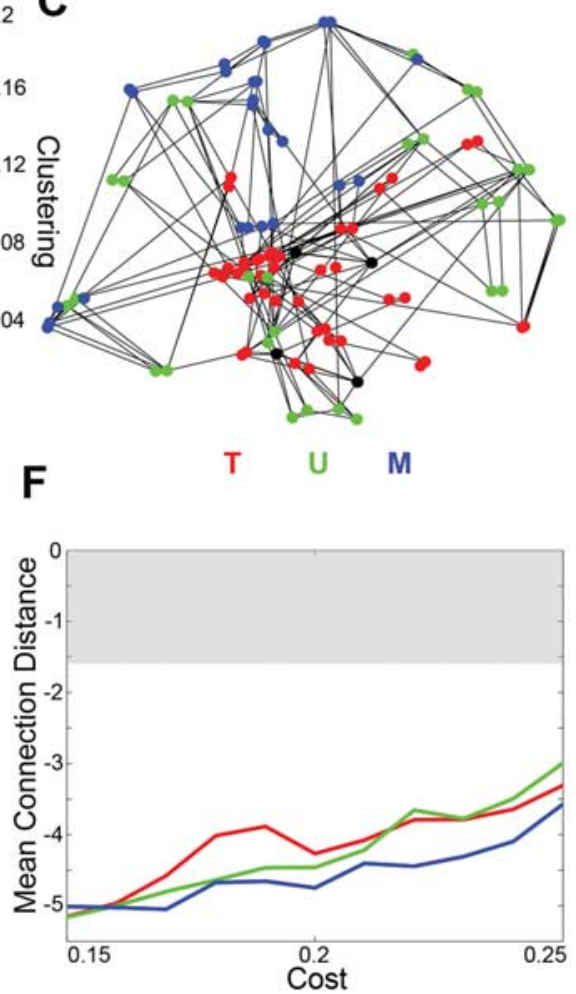

Figure 2. Organization of normal human brain anatomical networks in the small-world regime. $A$, Small-world metric, $\sigma$, in the cost range $0.05<K<0.25$ showing that the mean value of $\sigma \geq$ 1.2 when $K<0.25$. $\boldsymbol{B}$, Minimum degree $k$ (red) and minimum clustering $C$ (black) of the whole-brain networks as functions of cost showing that clustering or degree of some nodes is 0 when $K<$ 0.15. C, Anatomical representation of a sparsely thresholded network showing regional nodes color coded according to their membership of classical cortical divisions: transmodal (red), unimodal (green), and multimodal (blue). Z-scores for hierarchy coefficients $(\beta$; in $\boldsymbol{D})$, degree correlation or assortativity $(r$; in $\boldsymbol{E}$ ), and mean connection distance ( $($; in $\boldsymbol{F}$ ) for the three cortical networks as a function of cost in the small-world regime; gray areas indicate the $80 \%$ confidence interval for the parameters estimated in comparable random graphs $(-1.65<Z<1.65)$.

As shown in Figure 2, we found that the sparsest networks were clearly small-world, e.g., $\sigma$ was $>2$ when only $5 \%$ of possible connections were represented in the network $(K \sim 0.05)$. However, as expected, when the connection cost was increased, the value of $\sigma$ monotonically declined toward an asymptotic value of 1 . For costs greater than $K \sim 0.25$, we found that $\sigma \leq 1.2$, indicating that the brain networks were becoming topologically indistinguishable from random graphs. Thus, we identified the range of costs $0.15 \leq K \leq 0.25$ as the regime associated with small-world properties of sparse but fully connected brain anatomical networks. All subsequent analysis focused on networks in this regime.

\section{Normal cortical network organization}

The small-world properties of the healthy human cortical network were consistent with previous reports (He et al., 2008). Moreover, the cumulative degree distribution of the whole-brain networks was found to follow an exponentially truncated power law, which was also consistent with previous studies of both anatomical (He et al., 2008) and functional (Achard et al., 2006) networks derived from (functional) MRI data (supplemental Fig. 1, available at www.jneurosci.org as supplemental material).

As shown in Table 2, regions of right premotor cortex (BA 6), orbitofrontal cortex (BA 11), middle temporal cortex (BA 21), retrosplenial (BA 29), dorsal lateral prefrontal cortex (BA 46), and insula (BA 14), were identified as hubs of the normal network by two of the four (degree or centrality) parameters considered; left retrosplenial cortex was identified by three parameters, and anterior cingulate cortex was identified as a hub by only one parameter.

\section{Network properties of normal cortical divisions}

The multimodal network $M$ was the only cortical division that demonstrated a significant degree of hierarchical organization: the mean hierarchy coefficient over all costs in the small-world regime was $\overline{\beta_{M}}=0.16$, and the maximum $Z$-score for the hierarchy coefficient of the multimodal network compared with random networks was $\max \left(Z_{M}(\beta)\right)=2.09, p \sim 0.04$. Unimodal $U$ and transmodal $T$ cortical network hierarchy coefficients were generally closer to $0\left(\overline{\beta_{U}}=-0.06 ; \overline{\beta_{T}}=-0.14\right)$ and consistently fell within the $80 \%$ confidence interval for the hierarchy coefficient of comparable random networks.

The transmodal network was the only cortical division that demonstrated a significant degree of assortativity: the mean assortativity over all costs in the small-world regime was $\overline{r_{T}}=0.048$, and the maximum $Z$-score for the assortativity of the transmodal network compared with random networks was $\max \left(Z_{T}(r)\right)=1.93$, $p=0.027$. Multimodal and unimodal networks had assortativity coefficients closer to zero $\left(\overline{r_{M}}=-0.015 ; \overline{r_{U}}=0.019\right)$ and consistently fell within the $80 \%$ confidence interval for assortativity of comparable random networks.

Mean connection distance $(d, \mathrm{~mm})$ was greatest for multimodal cortex $\left(\overline{d_{M}}=62\right)$, intermediate for unimodal cortex $\left(\overline{d_{U}}=\right.$ $59)$, and smallest for transmodal cortex $\left(\overline{d_{T}}=51\right)$. However, connection distance was significantly smaller for all cortical net- 
Table 2. Regional hubs of the multimodal cortical network

\begin{tabular}{lllll}
\hline & $B_{c}$ & $k$ & $C_{c}$ & $E_{c}$ \\
\hline $\begin{array}{l}\text { Healthy volunteers } \\
\text { R BA 6, }\end{array}$ & & & & \\
SuM & & & \\
R BA 11, & 2.9 & 2.4 & & \\
ObF & & & \\
R BA 21, & 2.9 & 2.1 & \\
MiT & & & \\
R BA 29, & 2.3 & 2.4 & \\
ReS & & & \\
R BA 46, & 2.5 & 2.4 & \\
DLpF & & & \\
L BA 14, & 2.5 & 2.2 & \\
Ins & & & \\
L BA 33, & 2.3 & 2.1 & \\
ACC \\
L BA 46,
\end{tabular}

Hubs are defined by various centrality parameters for healthy volunteers (HV) and people with schizophrenia (SCZ) Values indicate the number of SDs away from the mean. L, Left; R, right. See Table 1 for abbreviations of Brodmann's areas.

works than for comparable random networks $\left(\overline{z_{T}}=4.54, p=2 \times\right.$ $\left.10^{-6} ; \overline{z_{U}}=4.24, p=1 \times 10^{-5} ; \overline{z_{M}}=4.13, p=2 \times 10^{-5}\right)$.

In short, these results suggest that all cortical divisions share the important organizational principle of tending to reduce wiring costs (compared with random networks), but multimodal cortex is differentiated by significantly nonrandom hierarchy and transmodal cortex is differentiated by nonrandom assortativity of network organization.

\section{Cortical network organization in schizophrenia}

The whole-brain anatomical networks constructed from MRI data on people with schizophrenia also showed fully connected, small-world properties $(\sigma>1)$ in the cost range $0.15 \leq K \leq 0.25$. The degree distribution of the schizophrenic network also followed an exponentially truncated power law (supplemental Fig. 1, available at www.jneurosci.org as supplemental material).

However, as shown in Table 2, the hubs of the schizophrenic network were qualitatively different from the hubs of the normal network. For example, dorsolateral prefrontal cortex, which was identified bilaterally as a hub in healthy volunteers, did not show this property in patients. Instead, insula, thalamus, temporal pole, pars opercularis (inferior frontal), inferior temporal, and precentral cortex were identified as hubs by at least one parameter in the patient group. Only one region (inferior temporal gyrus; BA 37) was consistently identified as a hub in the schizophrenic network by all four centrality parameters.

In addition to its effects on global organization, we also con- sidered the effects of schizophrenia on organization of each of the three subdivisions of cortex: transmodal, unimodal, and multimodal. There were a number of differences between the groups in organization of the multimodal cortical network. As shown in Figure 3, the multimodal network was less hierarchical throughout the small-world regime in people with schizophrenia, and this difference was statistically significant (permutation test, $p=$ 0.018 ). The between-group differences in hierarchical organization of the multimodal cortical network were also visualized graphically (Fig. 4). Finally, the mean connection distance was significantly greater for the multimodal network in people with schizophrenia (permutation test, $p=0.028$ ).

All topological changes were specific to the multimodal network because there were no significant differences between groups in hierarchy, assortativity, or connection distance of transmodal or unimodal networks.

We finally explored group differences in the organization of the networks at a regional level by testing for a significant between-group difference in the degree, clustering, and betweenness centrality of each node separately (Fig. 3). The clustering coefficient was the most sensitive measure of between-group differences: 23 regional nodes showed significant differences in clustering, mostly (78\%) in the left hemisphere, and most $(61 \%)$ reflecting increased clustering in schizophrenia. The regions predominantly affected were in premotor, prefrontal, orbitofrontal, inferior temporal, medial temporal, cingulate, and insular cortex. Similar profiles of regional abnormality in the patients compared with controls were identified in terms of degree and betweenness centrality (Fig. 3).

\section{Discussion}

\section{Properties of healthy human cortical networks}

One of our main findings was that classical divisions of normal human cortex shared some general principles of large-scale anatomical organization but also differed in some important global network properties.

All cortical networks (multimodal, unimodal, and transmodal) demonstrated connection costs significantly less than expected in comparable random graphs. This is compatible with previous work highlighting the efficiency of (axonal) wiring in nervous systems and consistent with the hypothesis, first suggested by Durbin and Mitchison (1990), that minimization of connection costs has been an important fitness criterion for evolution of all major components of human brain (Chklovskii et al., 2002; Chklovskii, 2004). All three networks also shared smallworld properties and exponentially truncated power-law degree distributions (supplemental Fig. 1, available at www.jneurosci. org as supplemental material), implying the existence in each network of a subset of highly connected hub regions and replicating previous findings in healthy human whole-brain networks (He et al., 2008).

Small-worldness is an almost ubiquitous property of complex, real-life networks, and, more specifically, it makes sense as a principle of brain topology because it supports both modular and distributed (high complexity) processing dynamics, which are well recognized as complementary aspects of the computational repertoire of the brain (Sporns et al., 2002; Bassett and Bullmore, 2006). A truncated power-law degree distribution is another property that seems to be common to a wide variety of information systems, including the Internet and World Wide Web, the extent of truncation tending to be greater (the probability of a very high degree hub being smaller) in physically embedded networks, such as the global air transportation network. Our obser- 

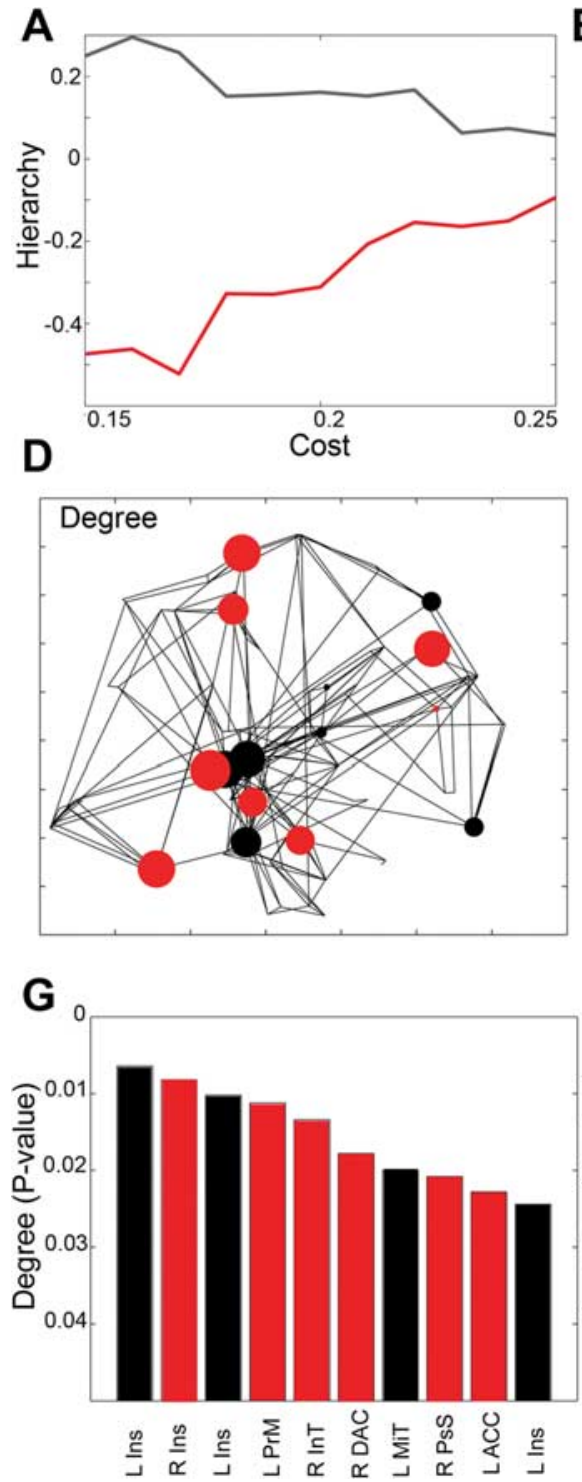

B
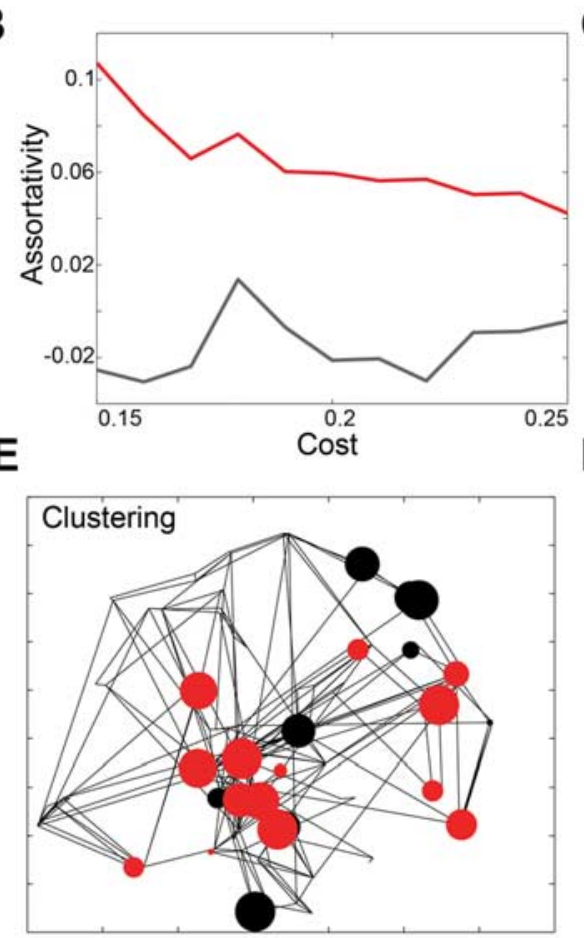

H

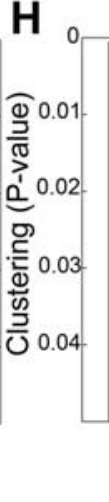

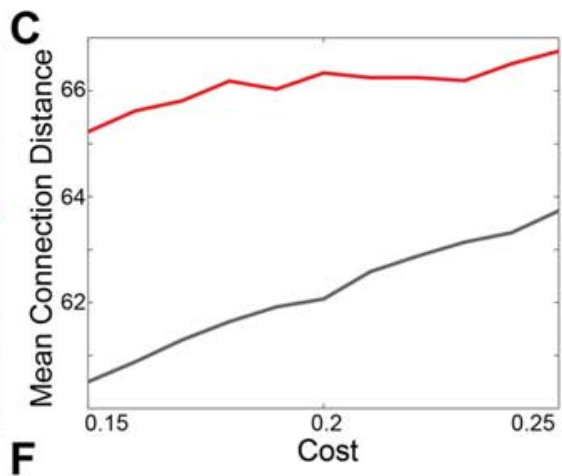

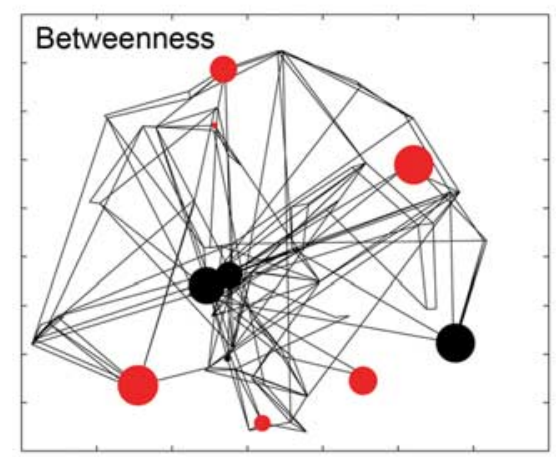

Figure 3. Effects of schizophrenia on organization of the multimodal cortical network. Top row, Hierarchy coefficients $(\boldsymbol{\beta}$; in $\boldsymbol{A})$, degree correlation or assortativity $(r$; in $\boldsymbol{B})$, and mean connection distance ( $d$; in $C$ ) as functions of cost in the small-world regime for healthy volunteers (black lines) and people with schizophrenia (red lines). Middle and bottom rows, Between-group differences in regional degree $(k$ in $\boldsymbol{D}, \boldsymbol{G})$, clustering $(\boldsymbol{C} ;$ in $\boldsymbol{E}, \boldsymbol{H})$, and betweenness centrality $\left(B_{c} ;\right.$ in $\left.\boldsymbol{F}, \boldsymbol{I}\right)$. In the middle row, regional differences are represented anatomically in the context of a sparsely thresholded whole-brain network; red nodes have significantly greater hub criteria, and black nodes have significantly smaller hub criteria, in people with schizophrenia. The size of the node is inversely proportional to the $p$ value of the between-group difference. In the bottom row, the red and black bars represent the rank-ordered and anatomically labeled $p$ values for the 10 most significant between-group differences in each of the hub criteria.

vation of hub regions in multimodal, unimodal, and transmodal networks is compatible with several previous reports of hub regions in whole-brain anatomical networks and suggests that the degree distribution is a conserved and invariant parameter of brain network organization at global and divisional scales.

However, it was also evident that the three cortical systems were not topologically identical. The strongest contrast was between the multimodal network and the transmodal network, with the unimodal network tending to have less differentiated properties. For example, mean connection distance was greatest for multimodal cortex, least for transmodal cortex, and intermediate for unimodal cortex. This is not surprising when we recall that many of the more recently evolved regions of neocortex comprising the multimodal system are anatomically distributed over the lateral surfaces of the cerebral hemispheres, whereas many of the phylogenetically older regions of allocortex or paleo- cortex comprising the transmodal system are concentrated in medial temporal and other limbic structures. A less predictable difference between the networks was that multimodal cortex was hierarchical, whereas transmodal cortex was assortative.

The hubs of a hierarchical network are highly connected but have low clustering; they are mainly connected to nodes that are not otherwise connected to each other. This architecture seems generally typical of corporate, information, or infrastructural systems; it favors executive-report relationships between nodes and conserves wiring costs, but it is vulnerable to targeted attack on the hubs (Ravasz and Barabasí, 2003; Sakata et al., 2005; Vahdatpour et al., 2005). The hubs of an assortative network are highly connected to each other, and this organization is more typical of social networks (although also reported for protein-protein networks) (Newman, 2002; Ravasz and Barabasí, 2003; Barrat et al., 2004; Trusina et al., 2004; Aftabuddin and Kundu, 2007). Assor- 
tative networks are robust against random or targeted removal of hubs, percolate easily, and have low epidemic thresholds. Different growth processes have been implicated in the generation of hierarchical and assortative networks (Cantanzaro et al., 2004).

Returning to our results in this context, we can see that the topological differences between multimodal and transmodal cortex could be significant in terms of their differential function, robustness to damage or disease, and development. More specifically, they suggest that multimodal and transmodal systems have been formed by different growth processes and/or evolved to satisfy different selection criteria. The emergence of a hierarchical organization in multimodal cortex, for example, might reflect a greater selection pressure on wiring cost at the expense of robustness as a function of the considerable increase in total brain volume associated with the evolutionary expansion of neocortex.

\section{Disorganization of cortical networks in schizophrenia}

This study represents the first effort to characterize the anatomical (dis)organization of brain networks in schizophrenia using graph theoretical tools, and, in so doing, we have observed a number of interesting results.

First, we have shown that several global parameters of whole-brain network architecture, such as smallworldness and the degree distribution, were close to their values in healthy volunteers, suggesting that key aspects of brain organization are highly conserved even in the presence of a putative neurodevelopmental disorder with profound effects on complex brain functions.

Second, we found that the three classical cortical divisions were differentially associated with the state of schizophrenia. Transmodal and unimodal cortical networks showed no significant difference in hierarchy, assortativity, or connection distance compared with healthy volunteers, whereas the multimodal cortical network had significantly reduced hierarchy and increased connection distance, as well as a trend to increased assortativity. In short, the multimodal network in schizophrenia had characteristics that might be interpreted as less efficiently wired, and the hubs of the network tended to be abnormally clustered and connected to other nodes of high degree. When we considered the multimodal network at a finer-grained regional level of topological analysis, these observations were corroborated by the finding that the predominantly prefrontal hubs of the normal network were replaced by inferior temporal, insular, and cingulate hubs in people with schizophrenia. Moreover, there were numerous between-group differences in regional clustering and other properties, involving mainly components of frontal, medial temporal, cingulate, and insular cortex, many of which have been suggested previously to be anatomically abnormal in schizophrenia (Weinberger et al., 2001; Ellison-Wright et al., 2008).

In short, our data are consistent with previous evidence that hubs are less prominent.
B

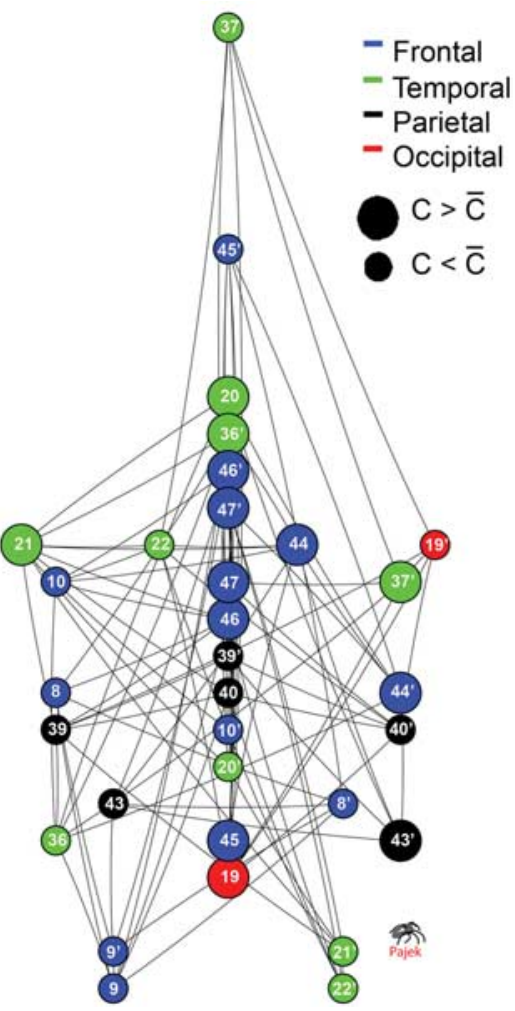

Figure 4. Graphical visualization of multimodal network hierarchy in healthy volunteers $(\boldsymbol{A})$ and people with schizophrenia the bottom of the normal hierarchy, which is dominated by highly connected nodes (many of them frontal) with low clustering;

schizophrenia may be conceived of as a dysconnectivity syndrome (Volkow et al., 1988; Weinberger et al., 1992; Bullmore et al., 1997; Meyer-Lindenberg et al., 2001, 2005), principally impacting on the normally efficient constitution of a frontally dominated hierarchical network of multimodal cortex. This view is consistent with many previous cognitive studies of schizophrenia, which have repeatedly emphasized deficits in executive and attentional processes dependent on lateral prefrontal cortical connectivity (Weinberger et al., 2001) and the many previous reports of functional and structural MRI abnormalities in multimodal association cortex and related white matter tracts (Woodruff et al., 1997; Weinberger et al., 2001; Buchanan et al., 2004; Honea et al., 2005; Kanaan et al., 2005; Gur et al., 2007; Kubicki et al., 2007). Our data are also potentially consistent with theoretical models of abnormal anatomical connectivity in schizophrenia at a synaptic level (Friston, 1998). For example, abnormal experience-dependent plasticity, leading to changes in synaptic density or dendritic arborization, could be reflected by abnormal inter-regional covariation of gray matter volume, even in the absence of macroscopic abnormalities of white matter tracts detectable by diffusion tensor imaging (DTI).

\section{Methodological issues}

The main limitation of our analysis is that, because the anatomical connectivity matrix is estimated on the basis of inter-regional correlations estimated over subjects, we have no opportunity to directly relate network metrics to individual differences in brain functions. 
It is also notable that our measurements of regional gray matter volume and the definition of multimodal, transmodal, and unimodal systems were both based on a priori anatomical classifications that are well respected and broadly conventional but to which alternatives exist. Adoption of different anatomical standards may have some effect on the pattern of results, but, pending a consensus on human cortical parcellation, this issue will remain somewhat arbitrary.

Another important consideration is that we have not made direct measurements of anatomical connectivity, as can be done by tract tracing in animals, but have instead inferred anatomical connectivity from covariation in regional gray matter volume. A direct proof that correlations of gray matter volume across subjects are indicative of axonal connectivity via white matter tracts has not been provided. However, strong correlations between brain regions known to be anatomically connected have been observed in previous optimized voxel-based morphometry studies (Mechelli et al., 2005; Pezawas et al., 2005). Similarly, correlations between left inferior frontal and superior temporal regions in cortical thickness measurements were validated previously as indirect markers of anatomical connectivity by direct comparison with DTI measurements of the arcuate fasciculus (Lerch et al., 2006). There are also hypothetical reasons to expect that interconnected regions might have correlated gray matter volumes attributable to the mutually trophic effects of connectivity on growth of connected regions. For example, neurotrophic factors such as BDNF, and glutamatergic signaling via the NMDA receptor, are known to promote neuronal survival as well as maintain and increase dendritic volume in the adult brain (Burgoyne et al., 1993; Monfils et al., 2004). However, we acknowledge that the cellular substrates of covariation of MRI measurements of gray matter volume have not been completely determined (Weinberger and McCure, 2002), so our inference of anatomical connectivity should be regarded currently as tentative.

It also is important to acknowledge that brain volume measurements made with MRI reflect aspects of brain biology other than neuronal elements, including blood volume, perfusion, and physiological measures related to tissue hydration, which could have implications for correlated volume measures (Weinberger and McCure, 2002). Antipsychotic medications and heavy smoking, which are relatively specific to the patient sample, have been shown to have effects on these non-neuronal components of brain volume, which could bear on group differences found in this study. In future work, these and other issues could perhaps be resolved by estimating the anatomical network individually for each subject using DTI or by directly investigating cellular correlates of altered gray matter (co)variation in MRI measurements of appropriate animal models.

\section{References}

Achard S, Bullmore ET (2007) Efficiency and cost of economical brain functional networks. PLoS Comput Biol 3:e17.

Achard S, Salvador R, Whitcher B, Suckling J, Bullmore E (2006) A resilient, low-frequency, small-world human brain functional network with highly connected association cortical hubs. J Neurosci 26:63-72.

Aftabuddin M, Kundu S (2007) Hydrophobic, hydrophilic, and charged amino acid networks within protein. Biophys J 93:225-231.

Barrat A, Barthélemy M, Pastor-Satorras R, Vespignani A (2004) The architecture of complex weighted networks. Proc Natl Acad Sci USA 101:3747-3752.

Bassett DS, Bullmore E (2006) Small-world brain networks. Neuroscientist $12: 512-523$

Bassett DS, Meyer-Lindenberg A, Achard S, Duke T, Bullmore ET (2006) Adaptive reconfiguration of fractal small-world human brain functional networks. Proc Natl Acad Sci U S A 103:19518-19523.
Bollobás B (1985) Random graphs. London: Academic.

Buchanan RW, Francis A, Arango C, Miller K, Lefkowitz DM, McMahon RP, Barta PE, Pearlson GD (2004) Morphometric assessment of the heteromodal association cortex in schizophrenia. Am J Psychiatry 161:322-331.

Bullmore ET, Frangou S, Murray RM (1997) The dysplastic net hypothesis: an integration of developmental and dysconnectivity theories of schizophrenia. Schizophr Res 28:143-156.

Burgoyne RD, Graham ME, Cambray-Deakin M (1993) Neurotrophic effects of NMDA receptor activation on developing cerebellar granule cells. J Neurocytol 22:689-695.

Cantanzaro M, Caldarelli G, Pietronero L (2004) Assortative model for social networks. Phys Rev E 70:037101.

Chen BL, Hall DH, Chklovskii DB (2006) Wiring optimization can relate neuronal structure and function. Proc Natl Acad Sci USA 103:4723-4728.

Chen ZJ, He Y, Rosa-Neto P, Germann J, Evans AC (2008) Revealing modular architecture of human brain structural networks by using cortical thickness from MRI. Cereb Cortex, in press.

Chklovskii DB (2004) Exact solution for the optimal neuronal layout problem. Neural Comput 16:2067-2078.

Chklovskii DB, Schikorski T, Stevens CF (2002) Wiring optimization in cortical circuits. Neuron 34:341-347.

Durbin R, Mitchison G (1990) A dimension reduction framework for understanding cortical maps. Nature 343:644-647.

Egan MF, Goldberg TE, Gscheidle T, Weirich M, Bigelow LB, Weinberger DR (2000) Relative risk of attention deficits in siblings of patients with schizophrenia. Am J Psychiatry 157:1309-1316.

Ellison-Wright I, Glahn DC, Laird AR, Thelen SM, Bullmore E (2008) The anatomy of first-episode and chronic schizophrenia: an anatomical likelihood estimation meta-analysis. Am J Psychiatry 165:1015-1023.

Friston KJ (1998) The disconnection hypothesis. Schizophr Res 30: $115-125$.

Good CD, Johnsrude IS, Ashburner J, Henson RN, Friston KJ, Frackowiak RS (2001) A voxel-based morphometric study of ageing in 465 normal adult human brains. Neuroimage 14:21-36.

Gur RE, Keshavan MS, Lawrie SM (2007) Deconstructing psychosis with human brain imaging. Schizophr Bull 33:921-931.

He Y, Chen ZJ, Evans AC (2007) Small-world anatomical networks in the human brain revealed by cortical thickness from MRI. Cereb Cortex 17:2407-2419.

Hilgetag CC, Burns GA, O’Neill MA, Scannell JW, Young MP (2000) Anatomical connectivity defines the organization of clusters of cortical areas in the macaque monkey and the cat. Philos Trans R Soc Lond B Biol Sci 355:91-110.

Honea R, Crow TJ, Passingham D, Mackay CE (2005) Regional deficits in brain volume in schizophrenia: a meta-analysis of voxel-based morphometry studies. Am J Psychiatry 162:2233-2245.

Humphries MD, Gurney K, Prescott TJ (2006) The brainstem reticular formation is a small-world, not scale-free, network. Proc Biol Sci 273:503-511.

Kanaan RA, Kim JS, Kaufmann WE, Pearlson GD, Barker GJ, McGuire PK (2005) Diffusion tensor imaging in schizophrenia. Biol Psychiatry 58:921-929.

Kubicki M, McCarley R, Westin CF, Park HJ, Maier S, Kikinis R, Jolesz FA, Shenton ME (2007) A review of diffusion tensor imaging studies in schizophrenia. J Psychiatr Res 41:15-30.

Latora V, Marchiori M (2001) Efficient behavior of small-world networks. Phys Rev Lett 87:198701.

Lerch JP, Worsley K, Shaw WP, Greenstein DK, Lenroot RK, Giedd J, Evans AC (2006) Mapping anatomical correlations across cerebral cortex (MACACC) using cortical thickness from MRI. Neuroimage 31:993-1003.

Mechelli A, Friston KJ, Frackowiak RS, Price CJ (2005) Structural covariance in the human cortex. J Neurosci 25:8303-8310.

Mesulam MM (1998) From sensation to cognition. Brain 121:1013-1052.

Meyer-Lindenberg A, Poline JB, Kohn PD, Holt JL, Egan MF, Weinberger DR, Berman KF (2001) Evidence for abnormal cortical functional connectivity during working memory in schizophrenia. Am J Psychiatry 158:1809-1817.

Meyer-Lindenberg AS, Olsen RK, Kohn PD, Brown T, Egan MF, Weinberger DR, Berman KF (2005) Regionally specific disturbance of dorsolateral 
prefrontal-hippocampal functional connectivity in schizophrenia. Arch Gen Psychiatry 62:379-386.

Monfils MH, VandenBerg PM, Kleim JA, Teskey GC (2004) Long-term potentiation induces expanded movement representations and dendritic hypertrophy in layer V of rat sensorimotor neocortex. Cereb Cortex 14:586-593.

Newman MEJ (2002) Assortative mixing in networks. Phys Rev Lett 89:208701.

Pezawas L, Verchinski BA, Mattay VS, Callicott JH, Kolachana BS, Straub RE, Egan MF, Meyer-Lindenberg A, Weinberger DR (2004) The brainderived neurotrophic factor val66met polymorphism and variation in human cortical morphology. J Neurosci 24:10099-10102.

Pezawas L, Meyer-Lindenberg A, Drabant EM, Verchinski BA, Munoz KE, Kolachana BS, Egan MF, Mattay VS, Hariri AR, Weinberger DR (2005) 5-HTTLPR polymorphism impacts human cingulate-amygdala interactions: a genetic susceptibility mechanism for depression. Nat Neurosci $8: 828-834$.

Ravasz E, Barabasí AL (2003) Hierarchical organization in complex networks. Phys Rev E 67:026112.

Sakata S, Komatsu Y, Yamamori T (2005) Local design principles of mammalian cortical networks. Neurosci Res 51:309-315.

Salvador R, Suckling J, Coleman MR, Pickard JD, Menon D, Bullmore E (2005) Neurophysiological architecture of functional magnetic resonance images of human brain. Cereb Cortex 15:1332-1342.

Sporns O, Tononi G, Edelman GM (2000) Theoretical neuroanatomy: relating anatomical and functional connectivity in graphs and cortical connection matrices. Cereb Cortex 10:127-141.

Sporns O, Tononi G, Edelman GM (2002) Theoretical neuroanatomy and the connectivity of the cerebral cortex. Behav Brain Res 135:69-74.

Sporns O, Honey CJ, Kötter R (2007) Identification and classification of hubs in brain networks. PLoS One 2:e1049.

Stephan KE, Hilgetag CC, Burns GA, O’Neill MA, Young MP, Kötter R
(2000) Computational analysis of functional connectivity between areas of primate cerebral cortex. Philos Trans R Soc Lond B Biol Sci 355:111-126.

Trusina A, Maslov S, Minnhagen P, Sneppen K (2004) Hierarchy measures in complex networks. Phys Rev Lett 92:178702.

Vahdatpour A, Tavakoli A, Falaki MH (2005) Hierarchical graph: A new cost effective architecture for network on chip. Lecture Notes Comput Sci 3824:311-320.

Volkow ND, Wolf AP, Brodie JD, Cancro R, Overall JE, Rhoades H, Van Gelder P (1988) Brain interactions in chronic schizophrenics under resting and activation conditions. Schizophr Res 1:47-53.

Watts DJ, Strogatz SH (1998) Collective dynamics of "small-world" networks. Nature 393:440-442.

Weinberger DR, McClure RK (2002) Neurotoxicity, neuroplasticity, and magnetic resonance imaging morphometry. Arch Gen Psychiatry 59:553-558.

Weinberger DR, Berman KF, Suddath R, Torrey EF (1992) Evidence of dysfunction of a prefrontal-limbic network in schizophrenia: a magnetic resonance imaging and regional cerebral blood flow study of discordant monozygotic twins. Am J Psychiatry 149:890-897.

Weinberger DR, Egan MF, Bertolino A, Callicott JH, Mattay VS, Lipska BK, Berman KF, Goldberg TE (2001) Prefrontal neurons and the genetics of schizophrenia. Biol Psychiatry 50:825-844.

Woodruff PW, Wright IC, Shuriquie N, Russouw H, Rushe T, Howard RJ, Graves M, Bullmore ET, Murray RM (1997) Structural brain abnormalities in male schizophrenics reflect fronto-temporal dissociation. Psychol Med 27:1257-1266.

Wright IC, Sharma T, Ellison ZR, McGuire PK, Friston KJ, Brammer MJ, Murray RM, Bullmore ET (1999) Supra-regional brain systems and the neuropathology of schizophrenia. Cereb Cortex 9:366-378.

Young MP (1992) Objective analysis of the topological organization of the primate cortical visual system. Nature 358:152-155. 